Please do not remove this page

RMIT

UNIVERSITY

\title{
On-the-fly GPS-based attitude determination using single- and double-differenced carrier phase measurements
}

Li, Yong; Zhang, Kefei; Roberts, Craig; Murata, M

https://researchrepository.rmit.edu.au/esploro/outputs/9921857966201341/filesAndLinks?institution=61RMIT_INST\&index=null

Li, Y., Zhang, K., Roberts, C., \& Murata, M. (2004). On-the-fly GPS-based attitude determination using single- and double-differenced carrier phase measurements. GPS Solutions, 8, 93-102.

https://doi.org/10.1007/s10291-004-0089-3

Document Version: Accepted Manuscript

Published Version: https://doi.org/10.1007/s10291-004-0089-3

Repository homepage: https://researchrepository.rmit.edu.au

(C) Springer-Verlag 2004

Downloaded On 2023/04/26 21:05:33 +1000 


\title{
On-the-fly GPS-based attitude determination using single- and double- differenced carrier phase measurements
}

\author{
Y. LI, K ZHANG AND C. ROBERTS \\ Dept of Geospatial Science, RMIT University, GPO Box 2476V, Melbourne 3001 VIC, \\ Australia
}

Tel: +61-3-9925-3351, Fax: +61-3-9663-2517, Email: yong.li@ rmit.edu.au

M. MURATA

Dept of Aerospace Engineering, National Defense Academy of Japan, Kanagawa, Japan

\begin{abstract}
Carrier phase measurements are primary observations for GPS attitude determination. Although the satelliterelated errors can be virtually eliminated by forming single differences, the baseline-related errors, such as line biases, are still present in the single-differenced carrier phase measurements. It is, therefore, difficult to resolve the single-differenced integer ambiguities due to the line biases. By forming double differences, the line biases of the single-differenced carrier phase measurements can be effectively removed. However, the main disadvantages of this method lie in the fact that the double-differenced measurements are mathematically correlated and consequently the attitude obtained from the double differences is noisy. This paper presents a new algorithm through which both single and double differences are used simultaneously to resolve these problems in real-time. The solution of the integer ambiguities can be obtained by searching for the most likely grid point in the attitude domain which is independent of the correlation with the double differences. Next, the line biases and corresponding single difference integer ambiguities can be resolved on-the-fly by using the noisy attitude solution obtained from the previous double difference procedure. In addition, the relationship between the physical signal path difference and the line bias is formed. A new method is also applied to derive the attitude angles through finding the optimal solution of the attitude matrix element. The proposed new procedure is validated using ground and flight tests. Results have demonstrated that the new algorithm is effective and can satisfy the requirement of real-time applications.
\end{abstract}

KEYWORDS: GPS, attitude determination, line bias, integer ambiguity resolution

\section{Introduction}

Recent research has demonstrated that the Global Positioning System (GPS) can play a key role in many applications, e.g. spacecraft attitude determination (AD) and navigation, due to its long-term stability, low cost and low power consumption (e.g. Fuller et al., 1997; Purivigraipong et al., 1999; Um and Lightsey, 2001; Reichert and Axelrad, 2001; Ziebart and 
Cross, 2003). Current GPS AD algorithms can generally be divided into three functional modules, namely line bias solution, integer ambiguity resolution (IAR) and attitude angular solution (Trimble Ltd, 1996). Line biases are mainly caused by the differences in cable lengths between antennas and the receiver (Cohen and Parkinson, 1992) or different radio frequency (RF) front ends in the receiver (Purivigraipong et al., 1999) or a combination of both. They are usually treated as constant variables and calibrated by a procedure prior to startup of a normal AD procedure in GPS attitude determination receivers, e.g. Trimble's TANS Vector (Trimble Ltd, 1996) and Space Systems/Loral's GPS Tensor (Fuller et al., 1997). Another method is that the line biases are treated as components of the state vector of the system, and therefore, estimated along with other state components (e.g. Ward and Axelrad,1996; Purivigraipong et al., 1999).

A GPS receiver can measure only the fractional part of the carrier phase. The integer number of wavelengths between antenna and satellite is unknown. This is the well-known integer ambiguity resolution problem. Two approaches have been developed to resolve the integer ambiguity problem for GPS-based attitude determination. The techniques are either motionbased (e.g. Cohen, 1996; Crassidis et al., 1999) or search-based (e.g. Quinn, 1993; Knight, 1994; Sutton, 1997). Motion-based methods need to collect data for a period of time during which obvious changes of a visible GPS constellation or the host platform rotation have occurred. The search-based methods use only single epoch measurements to find the most likely solution and these therefore occasionally are prone to incorrect solutions due to measurement noise. Two techniques have evolved. In the first technique, the search is carried out in a real number domain. The search space consists of all possible grid points of search parameters. These parameters can be the elevation and azimuth angles of a baseline (Caporali, 2001; Li et al., 2001) or the attitude angles of the host platform (Ziebart and Cross, 2003). In the second technique, the search is restricted to the integer number domain. The search space consists of all possible combinations of candidates of integer ambiguities (e.g. Quinn, 1993; Knight, 1994; Sutton, 1997).

The algorithms for attitude angular solution can also be roughly divided into the following two categories: (a) point estimation algorithms (e.g. Cohen, 1996; Crassidis and Markley, 1997; Bar-Itzhach et al., 1998; Li et al., 2002) and (b) stochastic filtering algorithms (e.g. Ward and Axelrad,1996; Chun and Park, 2001; Choukroun, D., 2002). There are two types of point estimation algorithms. The first type of point estimation algorithm uses vectorized observations (Crassidis and Markley, 1997; Bar-Itzhach et al., 1998) and can be considered as a two-level optimal estimation problem ( $\mathrm{Li}$ and Murata, 2001) — the least squares problem and Wahba's problem (Wahba, 1965). A number of algorithms for resolving the Wahba's problem have been proposed (i.e., Wertz, 1984; Mortari, 1998). The second type of point estimation algorithm deals with the differenced carrier phase measurements directly. It uses either a non-linear, least-square fit (NLLSFit) method (Cohen, 1996) or converts the problem equivalently into Wahba's problem (Cohen and Parkinson, 1992).

IAR is usually an initialization process since integer ambiguities are constant (assuming no cycle slips) and they do not need to be resolved again once they have been fixed. The IAR and attitude angular solution are therefore usually treated as two stand-alone procedures and they have been investigated separately in the literature (e.g., Cohen and Parkinson, 1992; Knight, 1994; Crassidis and Markley, 1997; Bar-Itzhach et al., 1998; Crassidis et al., 1999; Li et al., 2002).The line bias solution is coupled with the single-differenced IAR problem since the line biases remain in the single-differenced measurements. This means that it is necessary 
to carry out data pre-processing for the line bias solution when IAR is carried out in the single difference domain (i.e. Trimble Ltd., 1996). Although one can derive the solution in the double difference domain (i.e. Ziebart and Cross, 2003), the solution from the double differences is less accurate than the solution from the single differences.

This paper will combine all three modules for attitude determination in a compact form by incorporating the single and double differences to resolve the above problems. The main contributions in this paper are focused on the following aspects. First, a new algorithm that aims to find the optimal attitude matrix element solution (AMES) is used to derive the attitude angular solution. The AMES algorithm can be easily implemented and flexibly applied to an arbitrary configuration of antenna arrays ( $\mathrm{Li}$ et al., 2002). Second, the integer ambiguity solution in the single-differenced domain is obtained from the coarse attitude solution derived from the double-differenced measurements. The IAR procedure is carried out to search the attitude candidates in the double difference domain to avoid the problem caused by the line biases. This arrangement also avoids the correlation problem of the double differences (Hofmann-Wellenhof et al., 1997). Lastly, the relationship between the physical signal path difference and the line bias is formulated. A new algorithm for the point solution of line bias is presented.

This paper will first outline the proposed GPS AD procedure and describe its main operational modules. Then, algorithms for the attitude solution, the integer ambiguity resolution and the line bias estimation will be presented in detail. The results using the proposed procedure will be applied to the ground field tests and the flight experiments will be analyzed.

\section{GPS-Based Attitude Determination Procedure}

A GPS-based attitude determination system usually consists of at least two RF ports. Each port receives the GPS signals from an independent antenna. One can use two or more independent GPS receivers with $\mathrm{L}_{1}$ carrier phase output capability to construct an AD system (Caporali, 2001; Li et al, 2001). Due to the differences between receiver clocks, the betweenstation single-differenced carrier phase (SDCP) is not applicable to the derivation of the attitude solution, and the between-station between-antenna double-differenced carrier phase (DDCP) must be used in such systems. Most commercial products use a common reference clock to convert the received GPS RF signals into the intermediate frequency (IF) signals. IF signals will be then correlated to demodulate GPS data and generate observations such as pseudorange, Doppler, carrier phase and signal-to-noise ratio (SNR).

One benefit of using a common clock reference is that the clock error is the same for all RF signals including carrier phase measurements, and thus it can be removed by forming single differences. This is crucial for deriving the attitude solution from the SDCP (i.e., the carrier phase difference between the GPS signals received by two antennas separated by a short baseline). This kind of measurement also reflects the projection of the baseline vector onto the line-of-sight (LOS) vector to a GPS satellite. The solution derived from SDCP is more accurate than that derived from DDCP, since SDCP is less noisy. The SDCP measurement equation can be written as:

$$
\Delta \phi_{i j}=\hat{\mathbf{s}}_{i}^{T} \mathbf{A}^{T} \mathbf{b}_{j}-\Delta n_{i j} \cdot \lambda+\beta_{j}+v_{i j}
$$


where the subscript " $i$ " denotes the $i^{\text {th }}$ GPS satellite and " $j$ " denotes the $j^{\text {th }}$ baseline, and $\Delta \phi_{i j}$ is SDCP (in metres) associated with the $i^{\text {th }}$ satellite and the $j^{\text {th }}$ baseline, $\hat{\mathbf{s}}_{i}$ is the $i^{\text {th }}$ unit vector of LOS, $\mathbf{b}_{j}$ is the $j^{\text {th }}$ baseline vector, $\mathbf{A}$ is the 3 by 3 attitude matrix, $\Delta n_{i j}$ is the singledifferenced integer ambiguity associated with the $i^{\text {th }}$ satellite and the $j^{\text {th }}$ baseline, $\beta_{j}$ is the line-bias on the $j^{\text {th }}$ baseline, $\lambda$ is the wavelength of the GPS $\mathrm{L}_{1}$ carrier signal, and $v_{i j}$ is the measurement error of SDCP associated with the $i^{\text {th }}$ satellite and the $j^{\text {th }}$ baseline.

The line biases in equation 1 can be cancelled out by forming the double difference $(\nabla \Delta)$. The DDCP measurement equation can be written as:

$$
\nabla \Delta \phi_{i j}=\nabla \hat{\mathbf{s}}_{i}^{T} \cdot \mathbf{A}^{T} \mathbf{b}_{j}-\nabla \Delta n_{i j} \cdot \lambda+\zeta_{i j}
$$

where $\nabla \Delta \phi_{i j}$ is DDCP (in meters), $\nabla \hat{\mathbf{s}}_{i}$ is the difference of LOS vectors between the $i^{\text {th }}$ satellite and the reference satellite, $\nabla \Delta n_{i j}$ is the double-differenced integer ambiguity, $\zeta_{i j}$ is the measurement noise of DDCP.

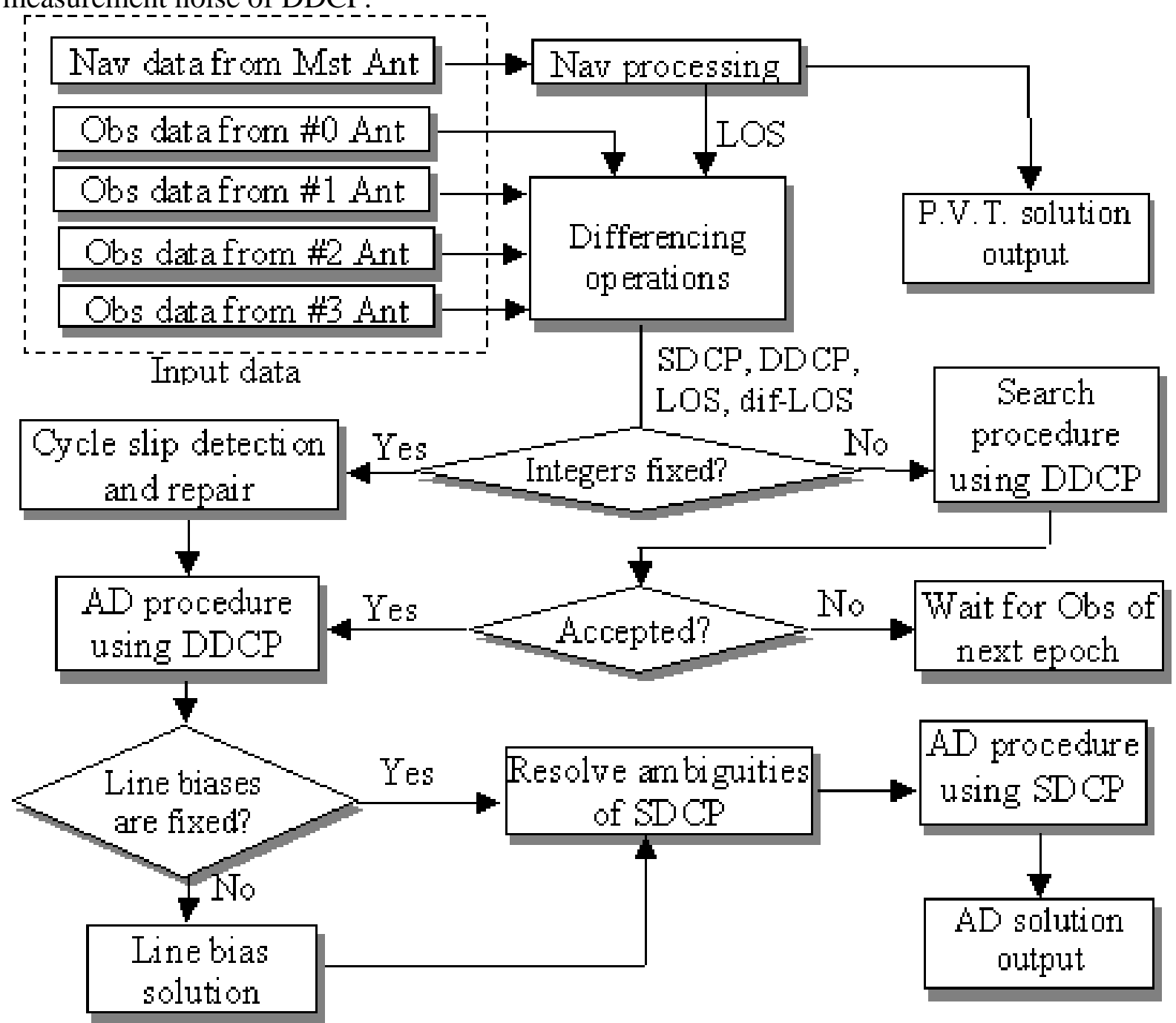

Figure 1. Flowchart of the proposed GPS attitude determination algorithm 
The proposed procedure for GPS attitude determination is presented in Figure 1. It uses both SDCP and DDCP data and provides the capability to estimate line-biases in real-time. The input data includes those for the positioning solution (termed as Nav data in Figure 1) and those for the attitude determination (termed as Obs data in Figure 1). The Nav processing block calculates position, velocity solution and outputs LOS to the AD procedure as well. The differencing operations including both single and double differences are carried out when the LOS and observation data become available. The IAR is used to fix integer ambiguities in the DDCP domain if integer ambiguities are not fixed. If integers have been fixed the processing will go to the "Yes" branch to check and repair cycle slips. The integer solution from the search procedure is further checked for acceptance or rejection. If the solution is rejected, the processing returns the start point and waits for observation and LOS data of the next epoch. Otherwise, if the solution is accepted, the processing begins to calculate the attitude angles. The modules for the line-bias solution and IAR in the SDCP domain run once the coarse attitude solution has been obtained from DDCP. The line-biases are no longer needed for calculation once they are filtered to sufficient accuracy. The final attitude solution is derived from the SDCP.

Any antenna in the array of the system can be used as the master antenna. Only Nav data from the master antenna is necessary, although those from other slave antennas are helpful in the processing. The position solution derived from the C/A code is accurate enough for attitude determination since actual position knowledge used in the AD processing derives from LOS vectors. This is based on the fact that the orbital height of GPS satellites is about $2 \times 10^{7} \mathrm{~m}$ and the overall C/A pseudorange error is less than $100 \mathrm{~m}$ (this is true since SA has been switched off), this introduces a relative error to LOS vectors at the level of $5 \times 10^{-6}$. This means only an error of about $5 \times 10^{-3} \mathrm{~mm}$ is introduced to the SDCP for a $1 \mathrm{~m}$ baseline. However the normal noise level of $\mathrm{L}_{1} \mathrm{SDCP}$ is about several millimeters (e.g. $5 \mathrm{~mm}$ in Cohen, 1996). Thus, the positioning error can be neglected in attitude determination.

\section{Algorithms}

\subsection{AMES Algorithm}

By taking into account the capability of tracking maneuvers, a point estimation algorithm rather than a filtering algorithm is used since a filtering mechanism may cause a delay in response to the maneuvers. The conventional point estimation algorithm is the NLLSFit method which uses an iterative procedure to obtain the potentially highest level of accuracy that a point estimation algorithm can achieve (Cohen, 1996). However, the inherent disadvantages of the NLLSFit method are that it needs a coarse attitude to initialize its timeconsuming iteration process. For real-time applications, a straightforward procedure is more acceptable, even if it would lose some accuracy. Based on this consideration, the AMES algorithm is adopted in this paper. Details of the AMES algorithm can be found in Li et al (2002) and is summarized hereafter.

A $(9 \times 1)$ state vector $\mathbf{a}$ to express the attitude matrix $\mathbf{A}$ is introduced in equation 3 ,

$\mathbf{a}=\left[\begin{array}{l:l:l}\mathbf{a}_{1}^{T} & \mathbf{a}_{2}^{T} & \mathbf{a}_{3}^{T}\end{array}\right]^{T}$

where $\mathbf{a}_{i}^{T}(i=1,2,3)$ is the $i^{\text {th }}$ row of $\mathbf{A}$. 
The cost function of the AD problem can then be written as follows,

$J(\mathbf{a})=\sum_{i=1}^{n} \sum_{j=1}^{m} w_{i j}^{2}\left(\Delta \phi_{i j}+\Delta n_{i j} \cdot \lambda-\beta_{j}-\mathbf{h}_{i j} \mathbf{a}\right)^{2}$

where $m$ is the number of baselines, and $n$ is the number of visible satellites, $w_{i j}^{2}$ is the weighted coefficient, and $\mathbf{h}_{i j}$ is a $(1 \times 9)$ matrix

$$
\mathbf{h}_{i j}=\left[\begin{array}{l:l:l}
b_{j x} \hat{\mathbf{s}}_{i}^{T} & b_{j y} \hat{\mathbf{s}}_{i}^{T} & b_{j z} \hat{\mathbf{s}}_{i}^{T}
\end{array}\right]
$$

where $b_{j x}, b_{j y}$ and $b_{j z}$ are the three components of vector $\mathbf{b}_{\mathbf{j}}$.

By introducing the following vectors

$$
\hat{\mathbf{u}}_{j}=\left(\sum_{i=1}^{n} w_{s i}^{2} \hat{\mathbf{s}}_{i} \hat{\mathbf{s}}_{i}^{T}\right)^{-1}\left[\sum_{i=1}^{n} w_{s i}^{2} \hat{\mathbf{s}}_{i}\left(\Delta \phi_{i j}+\Delta n_{i j} \cdot \lambda-\beta_{j}\right)\right]
$$

where $j=1,2, \ldots, m, w_{s i}^{2}$ is the weighted coefficient associated with the $i^{\text {th }}$ satellite, and $\hat{\mathbf{u}}_{j}$ is the solution of the $j^{\text {th }}$ baseline in the reference coordinate system.

The rows of $\mathbf{A}$ can be estimated separately as

$$
\hat{\mathbf{a}}_{i}=\sum_{j=1}^{m} w_{b j}^{2}\left(\mathbf{d}_{i}^{T} \mathbf{b}_{j}\right) \hat{\mathbf{u}}_{j}, i=1,2,3
$$

where $w_{b j}^{2}$ is the weighted coefficient associated with the $j^{\text {th }}$ baseline, and the vector of $\mathbf{d}_{i}$ is the $i^{\text {th }}$ row of the following $(3 \times 3)$ matrix

$$
\mathbf{D}=\left(\mathbf{B} \mathbf{W}_{b} \mathbf{B}^{T}\right)^{-1}
$$

where $\mathbf{W}_{b}$ is a $(3 \times 3)$ diagonal matrix with three diagonal elements of $w_{b j}^{2}(j=1,2,3)$ respectively. $\mathbf{B}$ is $3 \times m$ matrix which consists of $m$ baseline vectors.

The case that $\left(\mathbf{B} \mathbf{W}_{b} \mathbf{B}^{\mathrm{T}}\right)$ is singular usually implies that the antenna array is of a coplanar configuration. The weighted least squares solution for coplanar baseline configurations can be derived from equation 7 as follows,

$$
\hat{\mathbf{a}}_{i}=\frac{1}{e} \sum_{j=1}^{m} w_{b j}^{2}\left(e_{i 1} b_{j x}+e_{i 2} b_{j y}\right) \hat{\mathbf{u}}_{j}, i=1,2
$$

By taking into account the orthogonal property of the attitude matrix, the third row of $\mathbf{A}$ equals the cross product of the first and second rows of $\mathbf{A}$

$\mathbf{a}_{3}=\mathbf{a}_{1} \times \mathbf{a}_{2}$

where

$$
e_{11}=\sum_{j=1}^{m} w_{b j}^{2} b_{j y}^{2}
$$




$$
\begin{aligned}
& e_{12}=e_{21}=-\sum_{j=1}^{m} w_{b j}^{2} b_{j x} b_{j y} \\
& e_{22}=\sum_{j=1}^{m} w_{b j}^{2} b_{j x}^{2} \\
& e=e_{11} e_{22}-e_{12} e_{21}
\end{aligned}
$$

Equations $9 \mathrm{a}$ and $9 \mathrm{~b}$ are further applied to the analysis of the experimental data. Note that the AMES gives the constraint-free solution in which the orthogonal constraint is not taken into account. One can refer to the literature ( $\mathrm{Li}$ et al., 2001) for details of a procedure to orthogonalize the AMES solution.

\subsection{Attitude-Based Search Method}

An efficient algorithm to fix the integer ambiguities is crucial to the success of attitude determination. The search procedure herein is carried out in the DDCP domain since the line biases vanish in the DDCP. One can use the coarse attitude solution from the search procedure to calculate the SDCP integer ambiguities later. The search method used in this paper is based on the ambiguity resolution function (ARF) that can be parameterized as angles of both elevation and azimuth of a baseline vector (Caporali, 2001; Li et al., 2001). ARFbased algorithms for IAR can also be found in other geodetic applications (i.e., Han and Rizos, 1996).

For a GPS-based attitude determination system with a multi-baseline configuration, more than one ARF function has to be used to coordinate each baseline if ARF is parameterized on the angles of elevation and azimuth. A more efficient method for this situation is to define the ARF by using the attitude angles of the host platform as the parameters

$$
\operatorname{ARF}(\gamma, p, y)=\frac{1}{n \cdot m} \sum_{i=1}^{n} \sum_{j=1}^{m} \cos \frac{2 \pi}{\lambda}\left[\nabla \Delta \phi_{i j}-\nabla \hat{\mathbf{s}}_{i}^{T} \cdot \mathbf{A}^{T}(\gamma, p, y) \mathbf{b}_{j}\right]
$$

where the search parameters of $\gamma, p$ and $y$ represent three attitude angles of roll, pitch and yaw respectively. For the convenience of computer programming, the reference satellite in the DDCP is counted in the calculation of equation 11. This also avoids sorting the array of the DDCP again if the index of the reference satellite in the array changes.

Figure $2 \mathrm{a}$ is the pitch-yaw ARF mesh plot with $\gamma=0^{\circ}, p \in\left[-90^{\circ}, 90^{\circ}\right]$, and $y \in\left[-180^{\circ}, 180^{\circ}\right]$. The solution point is at $(\gamma, p, y)=\left(0^{\circ}, 0^{\circ}, 119^{\circ}\right)$. Figure $2(\mathrm{~b})$ depicts the contour plot with gradient directions (as the arrows indicated in the figure) within the area around the solution point that is $p \in\left[-30^{\circ}, 30^{\circ}\right], y \in\left[90^{\circ}, 150^{\circ}\right]$. It is also shown in this Figure how the points converge to the correct solution. The solution lies at the sharpest peak. 


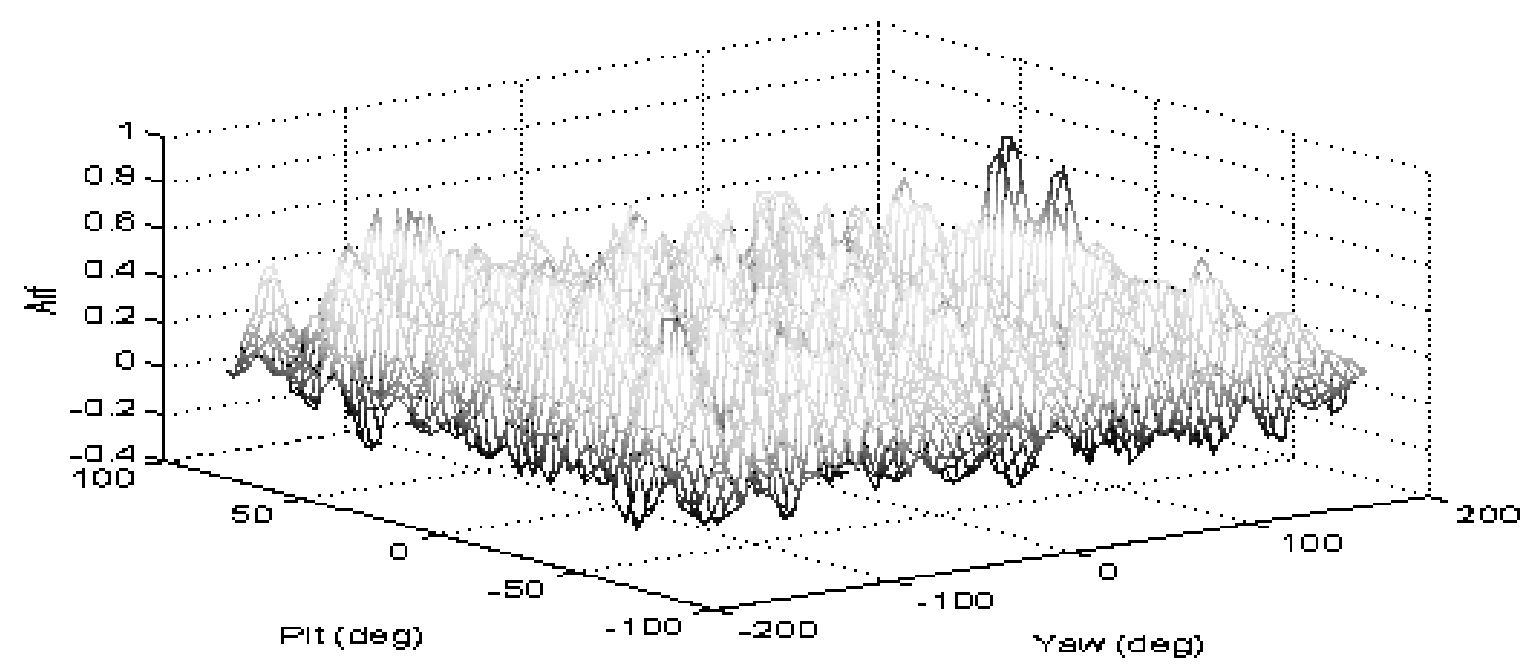

(a). Pitch-yaw ARF mesh plot $\left(\gamma=0^{\circ}\right)$

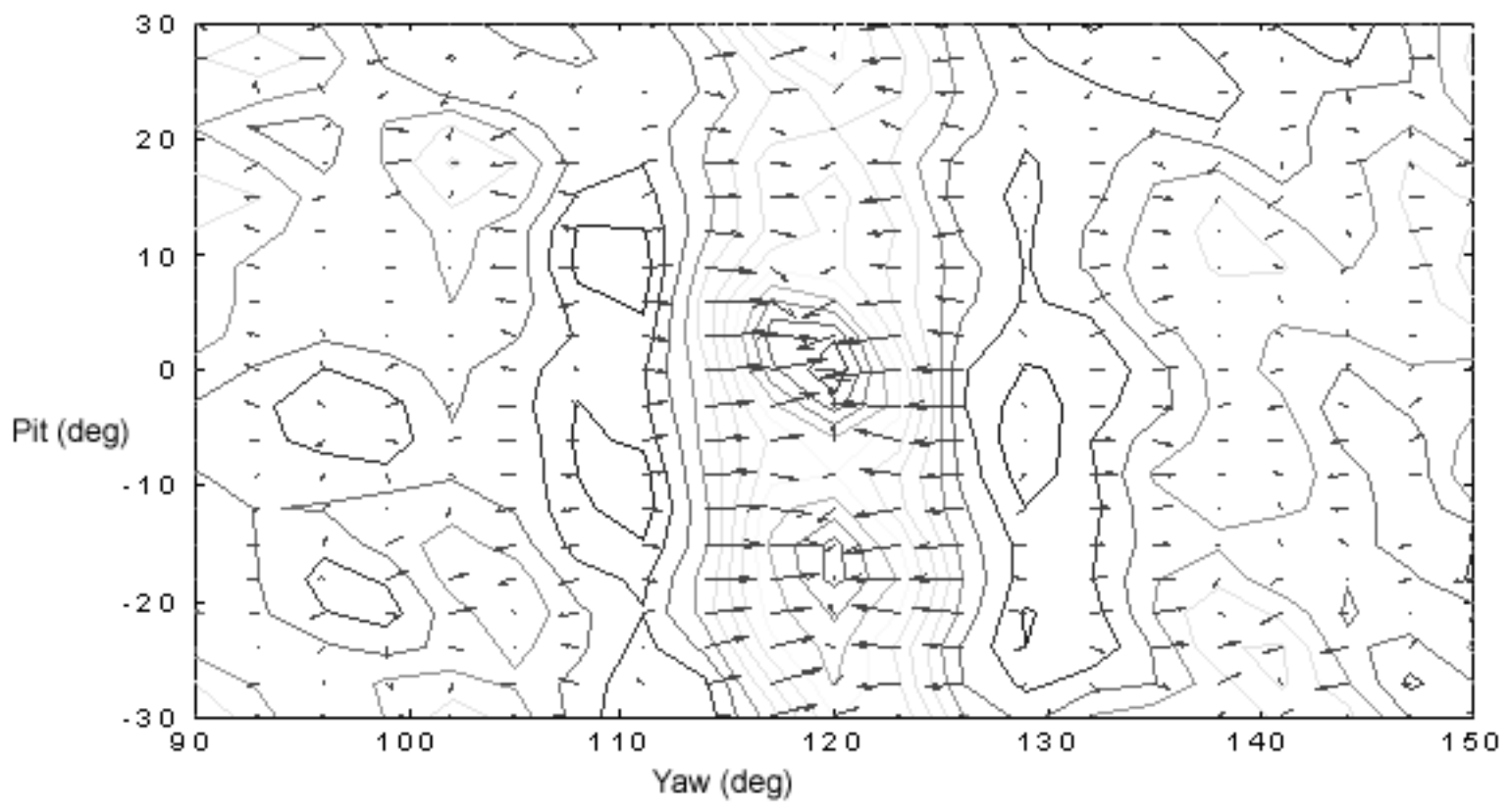

(b). Pitch-yaw ARF contour plot $\left(\gamma=0^{\circ}\right)$

Figure 2. The 2D and 3D plots of the pitch-yaw ambiguity resolution function

The search procedure based on equation 11 can be carried out by taking trials of all possible grid points for attitude angles. Without losing the generalization, suppose there are six satellites in view and three baselines in the structure. The redundancy of the attitude-based ARF search method is $12(5 \times 3-3)$ and the redundancy of the search based on the elevation and azimuth is 3 (5-2). Obviously, the attitude-based search procedure usually provides greater redundancy than the elevation-azimuth-based search procedure. This implies that the attitudebased search can give a more reliable solution from a statistical point of view. The efficiency of the search procedure takes advantages of both the high accuracy of the baseline length and 
the coarse attitude knowledge. However any carrier phase noise, multipath and other perturbations will potentially prevent the ARF search from achieving the correct solution.

Once the DDCP integer ambiguities have been fixed, a coarse attitude solution can be further derived from DDCP. This coarse solution is usually accurate enough to derive the line biases as well as to fix the SDCP integer ambiguities.

\subsection{Point Solution of the Line Bias}

If the line bias is treated only as a term in the mathematics regardless of its actual physical meaning it does not affect the processing of the attitude determination. However, further understanding the line bias physically can be helpful in deciding the quality of a solution of the line biases. Because the line biases are actually the phase biases, which reflect different lengths of signal paths between antennas and the receiver, the line bias on the $j^{\text {th }}$ baseline can be written as

$$
\beta_{j}=\left(\frac{2 \pi f_{L 1} \Delta l_{j}}{c}\right) \lambda=2 \pi \Delta l_{j}
$$

where $\Delta l_{j}$ is the difference in length of the two signal paths. The master path is from the master antenna to the RF integrated circuits (ICs), and the $j^{\text {th }}$ slave path is from the $j^{\text {th }}$ antenna to the RF ICs of the receiver. $f_{L 1}$ is the frequency of $\mathrm{L}_{1}$ carrier signal, and $c$ is the light speed in a vacuum.

Equation 12 reveals the relationship between the line bias and the actual difference of the signal paths. Furthermore, $\Delta l_{j}$ can be treated as the difference in length between the two RF cables which connect the master antenna and the $j{ }^{\text {th }}$ slave antenna to the receiver respectively if neglecting the differences that exist inside ICs of the receiver. For example, $\beta_{j}=3.806 \mathrm{~cm}$, from equation $12, \Delta l_{j}=\beta_{j} / 2 \pi \approx 6 \mathrm{~mm}$. This implies the length difference between the master cable and the $j^{\text {th }}$ cable is about $6 \mathrm{~mm}$.

It is easy to understand now that the values of the line biases change with the environmental temperature variation. The main reason is the environmental temperature variation causes different length variation of the cables. If the cables were made from the same materials, the difference would be very small as they share almost the same thermal characteristics. Therefore real-time line bias estimation capability becomes especially important for an AD procedure in some applications, i.e. space applications where temperature will vary greatly and frequently when spacecrafts go out of, or fall into, the shadow of the Earth. Unlike methods that treat the line biases as state variables to be estimated along with other attituderelated unknowns, a new approach is a standalone process to calculate the line biases as presented herein.

From equation 1, one can write down the line-bias for each $i$ and $j$ as

$$
\beta_{j}=\Delta \phi_{i j}-\hat{\mathbf{s}}_{i}^{T} \mathbf{A}^{T} \mathbf{b}_{j}+\Delta n_{i j} \cdot \lambda-v_{i j}
$$

Even if $\Delta n_{i j}$ is unknown at this step, one can still estimate $\beta_{j}$ by calculating its sine and cosine values after converting units into cycles instead of meters as follows: 
$\sin \hat{\beta}_{j}^{i}=\sin \frac{2 \pi}{\lambda}\left(\Delta \phi_{i j}-\hat{\mathbf{s}}_{i}^{T} \mathbf{A}^{T} \mathbf{b}_{j}\right)$

$\cos \hat{\beta}_{j}^{i}=\cos \frac{2 \pi}{\lambda}\left(\Delta \phi_{i j}-\hat{\mathbf{s}}_{i}^{T} \mathbf{A}^{T} \mathbf{b}_{j}\right)$

and then

$\hat{\beta}_{j}^{i}=\operatorname{tg}^{-1}\left(\frac{\sin \hat{\beta}_{j}^{i}}{\cos \hat{\beta}_{j}^{i}}\right) \cdot \lambda, \quad i=1,2, \ldots n$.

There are $n$ estimates of $\beta_{j}$ and the final solution is given as an average of $\hat{\beta}_{j}^{i}$ by

$\hat{\beta}_{j}=\frac{1}{n} \sum_{i=1}^{n} \hat{\beta}_{j}^{i}$

Note that line-bias values calculated by equation 16 are within one cycle, i.e.,

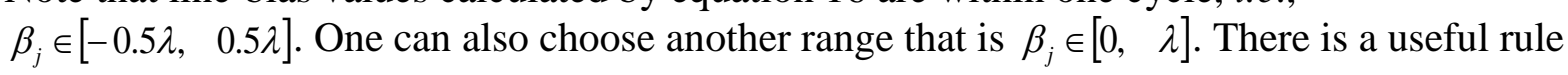
to decide which range of line-bias should be adopted. Because cables are almost the same length in a GPS AD system and usually these cables are made of the same material, the linebiases are always small and their values are closer to zero than to one cycle. Thus, one can

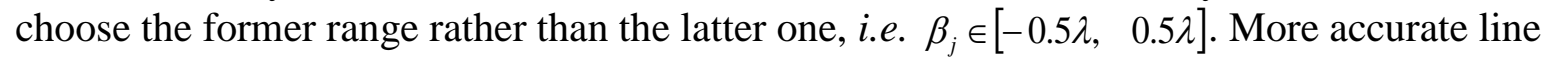
bias solution can be obtained by filtering this raw solution.

\section{Field Tests}

A number of field experiments have been conducted to validate the method proposed above. In the experiments, the raw single difference carrier phase measurements and LOS vectors were measured by a TANS Vector GPS receiver, which is a solid-state attitude-determination and position location system with a four-antenna array (Trimble Ltd., 1996).

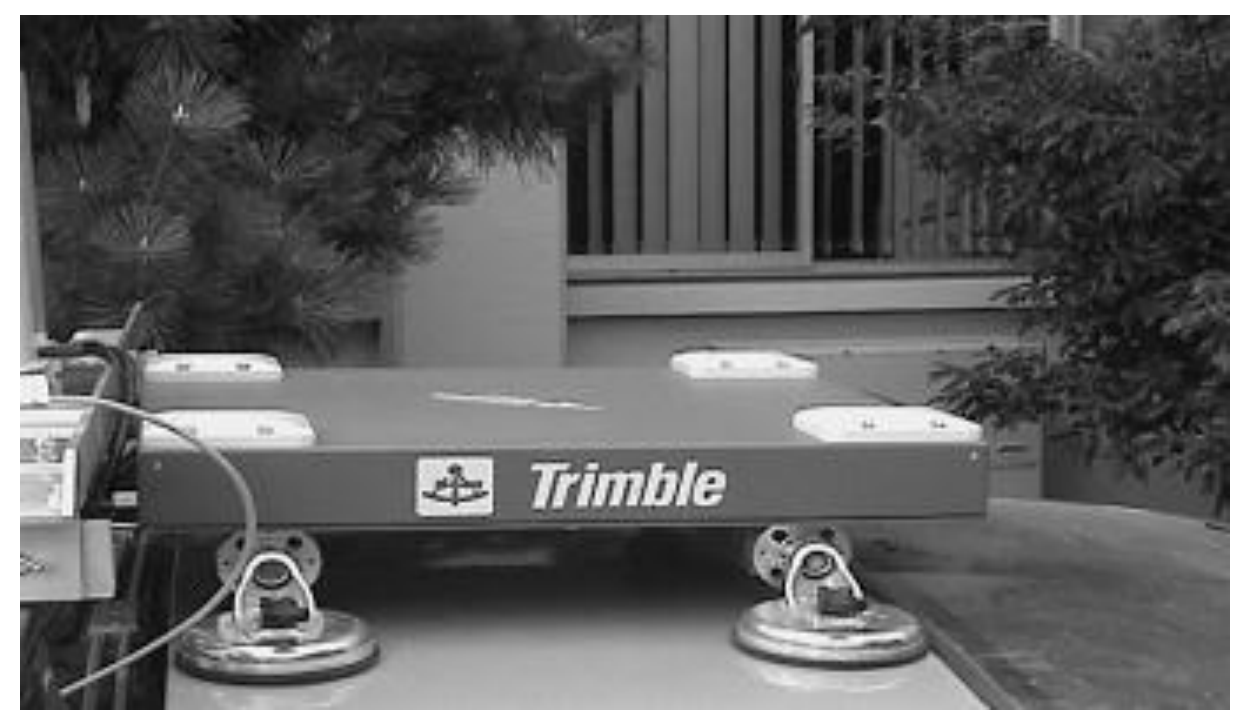

Figure 3. Trimble TANS Vector GPS receiver with four antennas 
Figure 3 shows a three-baseline configuration in which four antennas are arranged in a $41 \mathrm{~cm}$ by $41 \mathrm{~cm}$ square platform. The baselines can be expressed in the antenna coordinate system (or referred to the body frame system) as, $\mathbf{b}_{1}=\left[\begin{array}{lll}-l & l & 0\end{array}\right]^{T}, \mathbf{b}_{2}=\left[\begin{array}{lll}0 & 2 l & 0\end{array}\right]^{T}, \mathbf{b}_{3}=\left[\begin{array}{lll}l & l & 0\end{array}\right]^{T}$, where $l$ $=29 \mathrm{~cm}$.

The first experiment was conducted at Beijing Institute of Control Engineering on 23 December 1998. The receiver was positioned with a clear sky view and consistently recorded

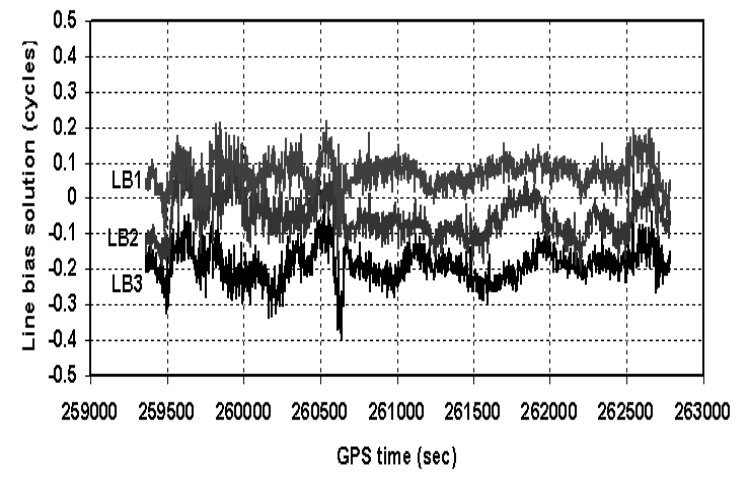

(a) Line bias solution

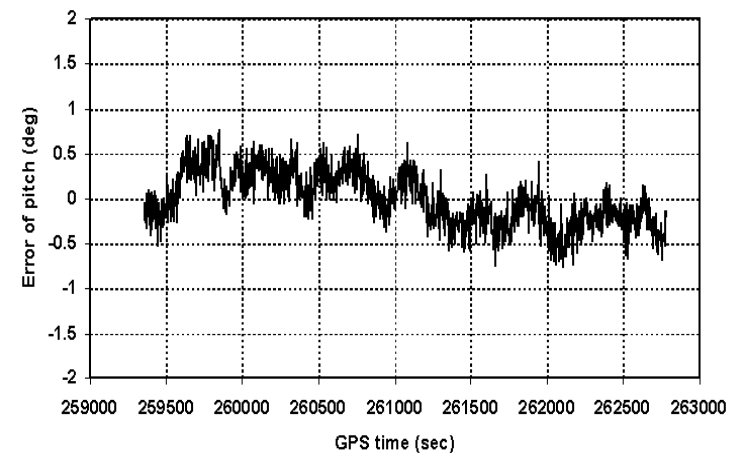

(c) Error of pitch

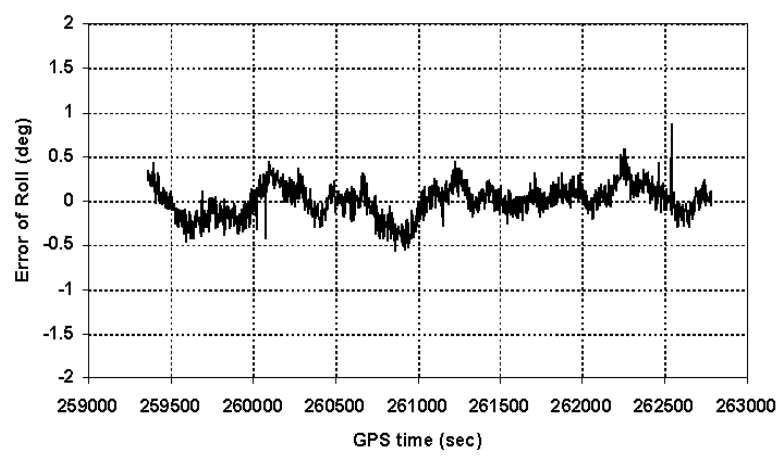

(b) Error of roll

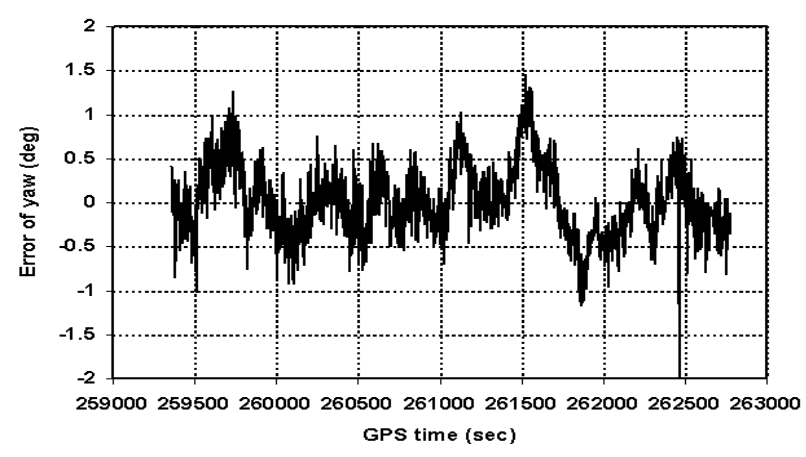

(d) Error of yaw

data for about one hour.

Figure 4. The line bias solution and the error of the attitude solution in the field test

Figure 4 shows the line bias solution versus GPS time. Average line biases are listed in Table 1 where the results come from two experiments that were carried out in the same conditions although different seasons (winter and summer respectively). It illustrates that the temperature variances due to environment show little difference on the line biases.

Table 1. Comparison of the line bias solutions in different seasons (in cycles)

\begin{tabular}{cccc}
\hline Line Biases & $\beta_{1}$ & $\beta_{2}$ & $\beta_{3}$ \\
\hline Winter (23/12/1998) & -0.200 & -0.083 & 0.064 \\
Summer (23/07/1999) & -0.149 & -0.071 & 0.074 \\
\hline \hline
\end{tabular}

Table 2. Evaluation of attitude solution error in the field test (in degrees)

\begin{tabular}{cccc}
\hline \hline Error of attitude solution & $\Delta$ Roll & $\Delta$ Pitch & $\Delta$ Yaw \\
\hline Standard deviation & 0.18 & 0.30 & 0.41 \\
Minimum & -0.56 & -0.76 & -2.17
\end{tabular}


The average attitude angles are $0.18^{\circ}$ in roll, $-0.98^{\circ}$ in pitch, and $-38.77^{\circ}$ in yaw respectively. The error of the attitude solution is listed in Table 2 and also shown in Figure 4. Suppose that the measurement noise is at the level of $5 \mathrm{~mm}$, an approximate and general rule of thumb for attitude determination angular accuracy (in radians) for a representative baseline length of $L$ (in meters) is given in (Cohen, 1996) as

$\sigma_{\theta}($ in radians $) \cong 0.005 / L$

This would introduce an angular error of about $0.3^{\circ}$ for a one-meter baseline. For the baseline configuration in the experiments, the longest baseline has a length of $0.58 \mathrm{~m}$. Thus according to equation 17, measurement noise would introduce an error of about $0.52^{\circ}$ to the attitude solution. From Table 2, it can be concluded that the AMES can achieve the nominal level of accuracy.

\section{Flight Tests}

The GPS receiver used in the tests was developed in the National Space Development Agency of Japan. The system has four RF ports and each port has eight channels. It can therefore track up to eight satellites simultaneously. Current configuration of the receiver unit contains a clock as a common reference to tag the time of the measurements of pseudorange, Doppler and carrier phase at a rate of $1 \mathrm{~Hz}$ (Li et al., 2003).

A number of flight experiments were conducted in November 2001. The GPS attitude unit was mounted on the body of the Dornier-228, National Aerospace Laboratory of Japan's aircraft, which is shown in Figure 5(a). The antennas were arranged to form an approximate $850 \mathrm{~mm}$ by $900 \mathrm{~mm}$ coplanar square configuration as shown in Figure 5(b). Baselines in the body frame are numerically defined as (in unit of meters), $\mathbf{b}_{1}=\{0,0.849,0\}, \mathbf{b}_{2}=\{-0.9,0,0\}$, and $\mathbf{b}_{3}=\{-0.9,0.853,0\}$.

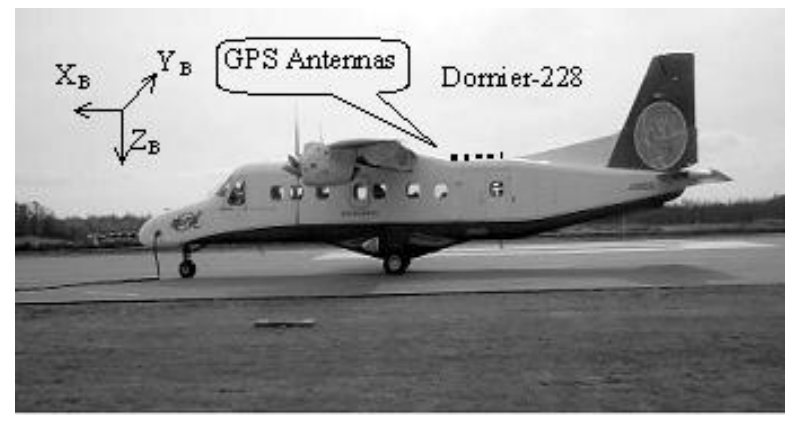

(a) Dornier-228 airplane

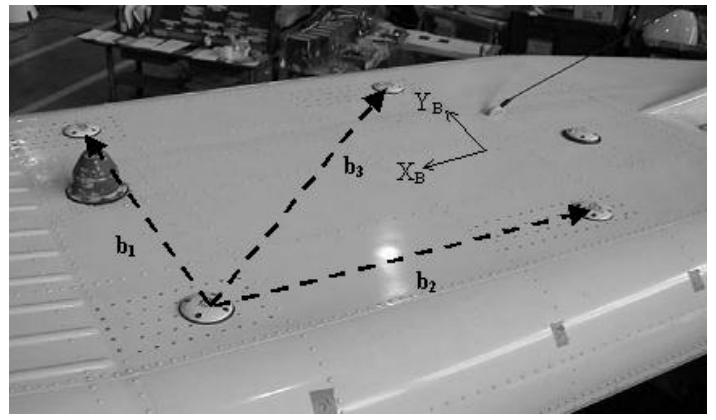

(b) Baseline configuration

Figure 5. GPS antenna mounting and baseline configuration in actual flight experiments

An IMU (inertial measurement unit) with three fiber optical gyros (FOG) and accelerometers were mounted on the aircraft to provide attitude reference to evaluate the GPS solution. The stability of FOG is, X-axis gyro: $0.08 \mathrm{deg} / \mathrm{hr}$; Y-axis gyro: $0.46 \mathrm{deg} / \mathrm{hr}$; and Z-axis gyro: 0.08 $\mathrm{deg} / \mathrm{hr}$ respectively. 


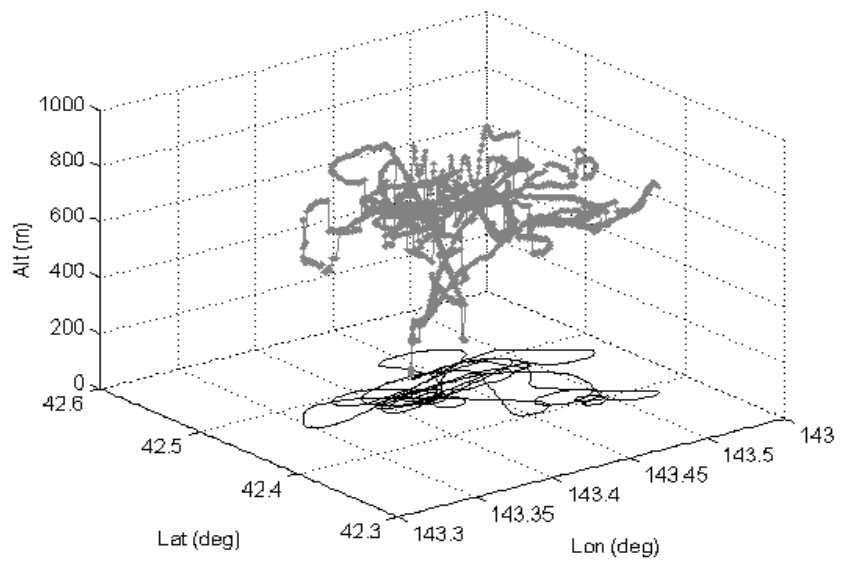

(a) 3D trajectory

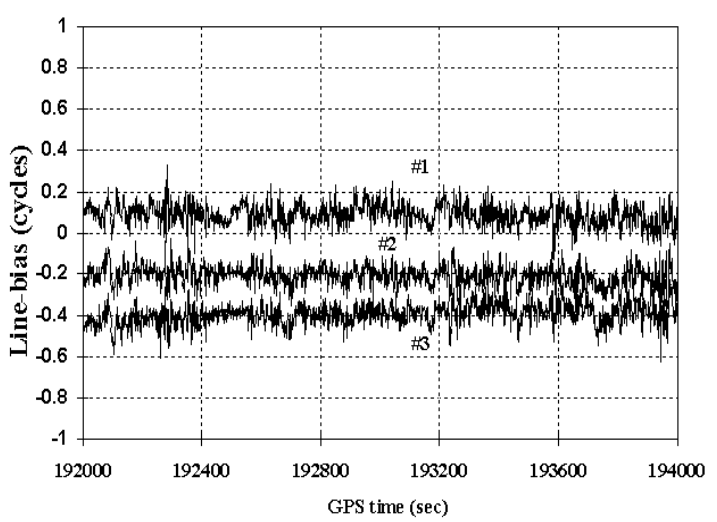

(b) On-the-fly line bias solution

Figure 6. The 3D trajectory of the flight experiment and line bias solution

The results of all flights demonstrate the efficiency of the algorithms, and the results of \#3 flight only are illustrated hereafter. The position and velocity are derived from the C/A code pseudorange measurements. The 3D trajectory is depicted in Figure 6(a) and the on-the-fly line bias solution is depicted in Figure 6(b). It illustrates that the performance of the algorithm is excellent in the experiments even during the maneuvers.

The attitude solution obtained from the GPS SDCP measurements by the AMES algorithm is shown in Figure 7. The differences between the AMES solution and the IMU output are presented in Figure 8. The average and standard deviation of the discrepancies between GPS and IMU are listed in Table 3. Both Table 3 and Figure 8 have shown that the AMES and IMU solutions are consistent. For the baseline configuration in the experiments, the longest baseline has a length of $1.24 \mathrm{~m}$. Thus according to Eq. (17), measurement noise would introduce an error of about $0.24^{\circ}$ to the attitude solution. From Table 3, it can be concluded that the AMES can achieve the nominal level of accuracy. Moreover, the results illustrate excellent performance of the AMES to track maneuvers and a fast computation speed.

Table 3. The average and standard deviation of the discrepancies between GPS and IMU solutions (unit: degrees)

\begin{tabular}{ccc}
\hline \hline Error of angles & Average & Standard deviation \\
\hline$\Delta$ Roll & 0.012 & 0.16 \\
$\Delta$ Pitch & -0.056 & 0.13 \\
$\Delta$ Yaw & -0.054 & 0.23 \\
\hline
\end{tabular}

The attitude-based ARF search method has successfully passed the flight tests for both on-line initialization and in-flight reset capability. Note that different search modes are considered in the procedure to deal with different situations that may occur in operation. These include initialization, operation reset, changes of satellites used in calculation and detection of cycle slips. All exhibit very satisfactory performance. 


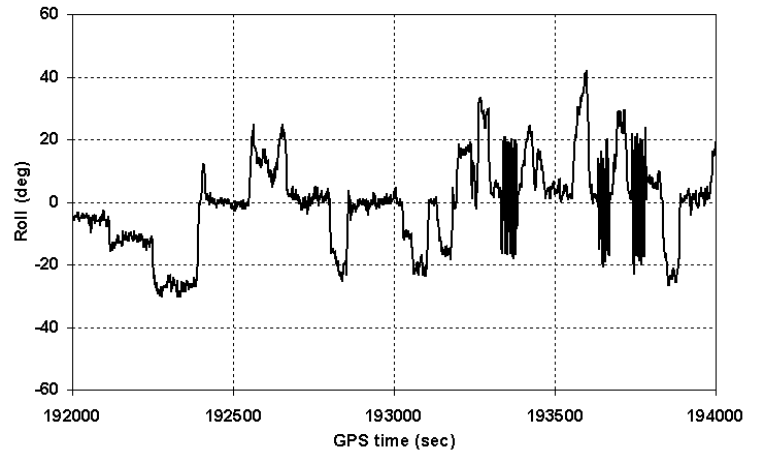

(7a) Roll

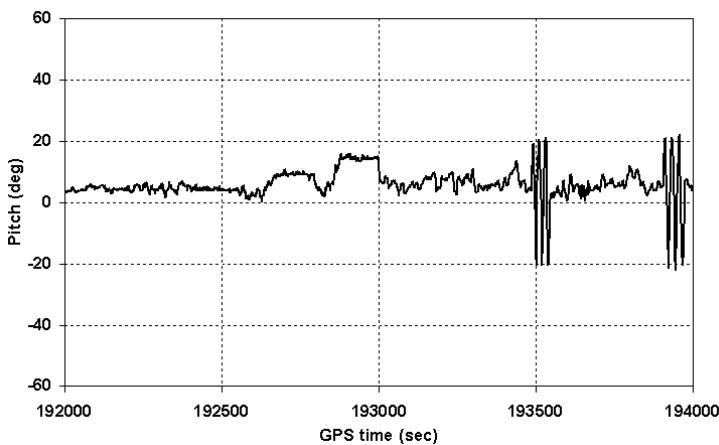

(7b) Pitch

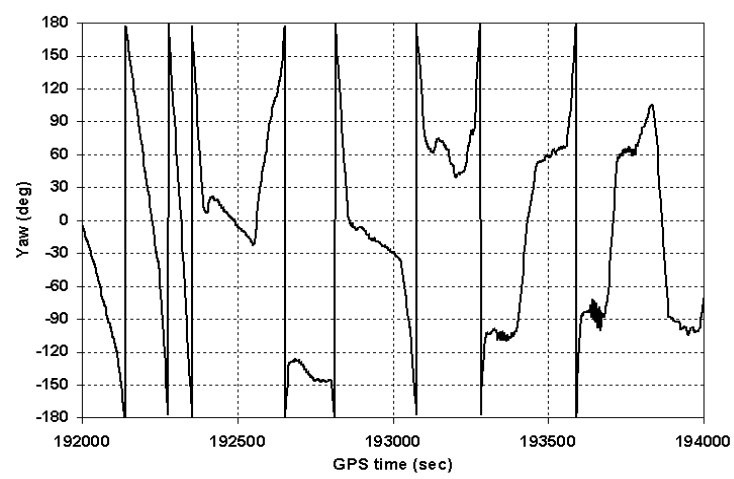

(7c) Yaw

Figure 7. GPS attitude solution (roll, pitch and yaw) vs. GPS time

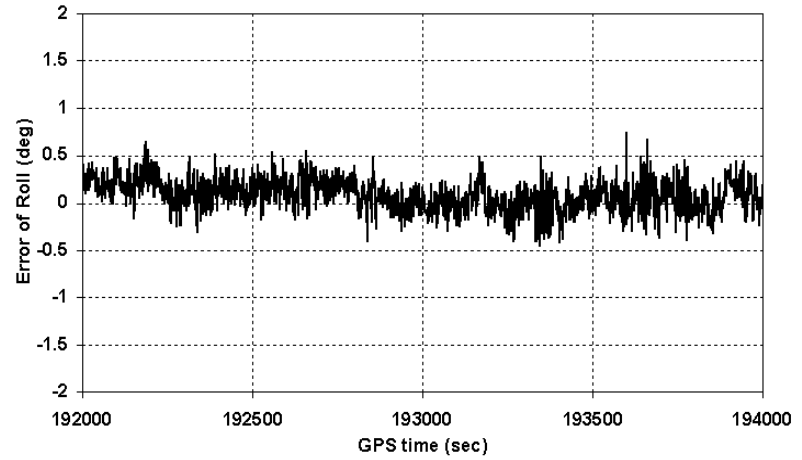

(8a) Error of Roll

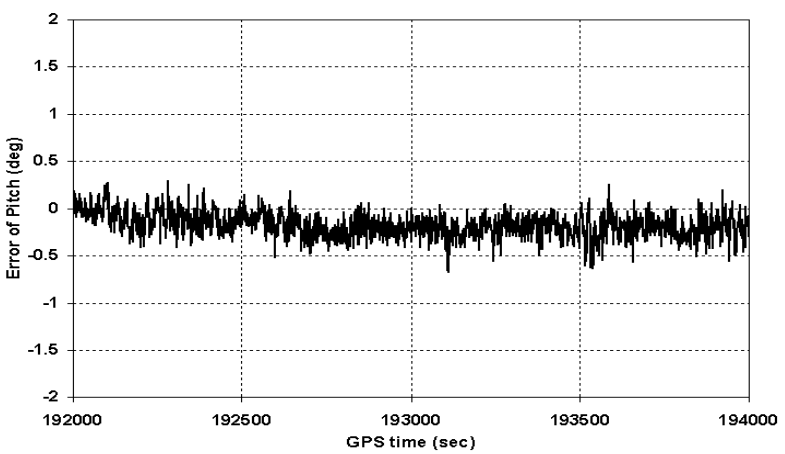

(8b) Error of Pitch

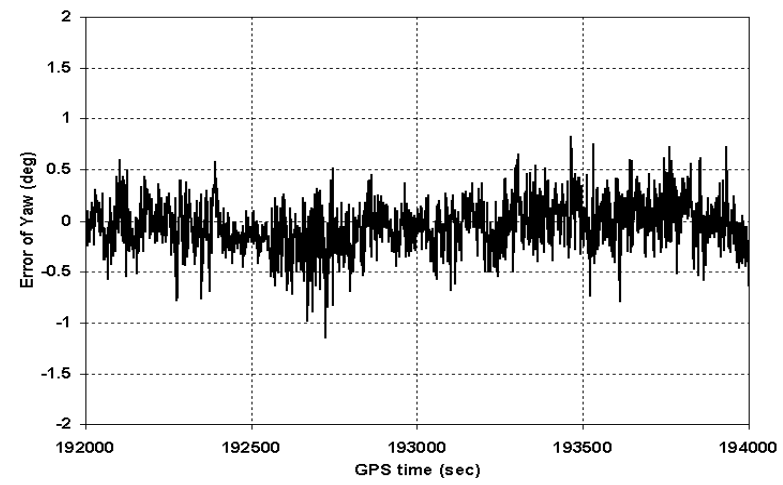

(8c) Error of Yaw

Figure 8. Difference between GPS attitude solution and output of IMU

\section{Conclusions}

This paper has presented a procedure for the GPS-based attitude determination in real-time through which both single- and double-differenced carrier phase measurements are used simultaneously. This method can overcome the problem incurred when only one type of measurement is used. This method can therefore isolate the line-bias problem from IAR in SDCP domain and obtain a much more accurate solution from SDCP instead of the noisy solution from DDCP. 
The paper has presented a series of new algorithms that resolve the main problems existing in the field of GPS attitude determination, i.e., problems for IAR, attitude solution and line-bias solution respectively. The experiments have demonstrated that the proposed procedure can provide a very reliable and efficient solution of the attitude of host platforms. The ARF is parameterized in the attitude angles and the search space is independent of the DDCP. Therefore the search, based on this ARF, can avoid the correlation problem which normally exists when searching for integer candidates.

The relationship between the line bias and the difference of physical signal paths has been formulated. An algorithm for the point solution of line biases has been presented and the experiments have demonstrated its high efficiency. This makes the configuration of the proposed procedure more flexible, i.e. the module for line bias solution is treated as a standalone functional block so that a prior calibration for line biases is no longer required.

The AMES algorithm can achieve the nominal accuracy that a GPS AD system can reach in the experiments. Its advantages such as excellent performance during maneuvers and fast computational speed were also demonstrated in the experiments. All of these features of AMES as well as its straightforward procedure make it very suitable for real-time applications.

\section{ACKNOWLEDGEMENTS}

We would like to acknowledge financial support from the Chinese government postdoctoral fellowship and the Japanese Science Technology Agency (STA) fellowship. The Beijing Institute of Control Engineering (BICE) is thanked for their provision of the TANS Vector's data and the National Aerospace Laboratory of Japan (NAL) and the National Space Development Agency of Japan (NASDA) for their permission to use the flight data in this paper. The authors acknowledge Mr. Baoxiang Sun and Mr. Yijun Gao at BICE, Mr. Yoshiyuki Ishijima at NASDA and Dr. Masatoshi Harigae at NAL for their help and cooperation.

\section{References}

Bar-Itzhach, I. Y., Montgomery, P.Y., Garrick, J.C. (1998). Algorithms for Attitude Determination Using the Global Positioning System, Journal of Guidance, Control and Dynamics, 21(6), 846-851.

Caporali, A. (2001). Basic Direction Sensing with GPS, GPS World, 12 (3), 44-50.

Choukroun, D. (2002). A Novel Quaternion Kalman Filter Using GPS Measurements, Proceedings of ION GPS2002 (pp.1117-1128), Alexandria, VA: Institute of Navigation.

Chun, C., Park, F.C. (2001). Dynamics-Based Attitude Determination Using the Global Posistioning System, Journal of Guidance, Control, and Dynamics, 24(3), 466-473.

Cohen, C.E., Parkinson, B.W. (1992). Integer Ambiguity Resolution of the GPS Carrier for Spacecraft Attitude Determination, Advances in the Astronautical Sciences, Vol. 78, 891-118. 
Cohen, C.E. (1996). Attitude Determination, In: Parkinson, B.W., Spilker, J.J. (eds) Global Positioning System, Theory and Applications, Vol. II, AIAA, Washington, DC, 519-538.

Crassidis, J.L., Markley, F.L. (1997). New Algorithm for Attitude Determination Using Global Positioning System, Journal of Guidance, Control, and Dynamics, 20(5), 891-896.

Crassidis, J.L., Markley, F.L., and Lightsey, E.G. (1999). Global Positioning System Integer Ambiguity Resolution Without Attitude Knowledge, Journal of Guidance, Control, and Dynamics, 22(2), 212-218.

Fuller, R., Hong, D., Hur-Diaz, S., Rodden, J., Tse, M. (1997). GPS Tensor ${ }^{\text {TM}: ~ A n ~ A t t i t u d e ~ a n d ~ O r b i t ~}$ Determination System for Space, Proceedings of ION GPS-97 (pp.299-311), Alexandria, VA: Institute of Navigation.

Han, S., Rizos, C. (1996). Improving the Computational Efficiency of the Ambiguity Function Algorithm, Journal of Geodesy, 70: 330-341.

Hofmann-Wellenhof, B., Lichtenegger, H. and Collins, J. (1997). Global Positioning System: Theory and Practice, Fourth, revised edition, Springer Wien New York, 191-196.

Knight, D. (1994). A New Method of Instantaneous Ambiguity Resolution, Proceedings of ION GPS-94 (pp. 707-716), Alexandria, VA: The Institute of Navigation.

Li, Y., Murata, M.(2001). A Two-Level Optimal Estimator for Attitude Determination Using GPS Measurements, Preprints of 15th IFAC Symposium on Automatic Control in Aerospace (pp. 235 - 240), September, Bologna/Forlì, Italy.

Li, Y., Nakajima, A., Murata, M., Isobe, T. (2001). Attitude Determination Using Two GPS Receivers for Antenna Control, Proceedings of the 45th Space Sciences and Technology Conference (pp. 1173 - 1178), October, Hamamatsu, Japan.

Li, Y., Murata, M., Sun, B. (2002). New Approach to Attitude Determination Using GPS Carrier Phase Measurements, Journal of Guidance, Control and Dynamics, 25(1), 130-136.

Li, Y., Murata, M., Ishijima, Y. (2003). Flight evaluation of New Algorithms for GPS Attitude Determination, Proceedings of SatNav 2003, The 6th International Symposium on Satellite Navigation Technology Including Mobile Positioning \& Location Services (Paper No. 58), July, Melbourne, Australia.

Mortari, D. (1998). Euler-q Algorithm for Attitude Determination from Vector Observations, Journal of Guidance, Control, and Dynamics, 21(2), 328-334.

Purivigraipong, S., Hashida, Y., Unwin, M.J. (1999). GPS Attitude Determination for Microsatellites, , Proceedings of ION GPS-99 (pp.2017-2026), Alexandria, VA: Institute of Navigation.

Quinn, P. G. (1993). Instantaneous GPS Attitude Determination, Proceedings, Proceedings of ION GPS-93 (pp. 603-615), Alexandria, VA: Institute of Navigation.

Reichert, A.K., Axelrad, P. (2001). Carrier-Phase Multipath Corrections for GPS Based Satellite Attitude Determination, Navigation - Journal of The Institute of Navigation, 48(2), 77-88.

Trimble Navigation Limited (1996). TANS Vector Specification and User's Manual, Software Version 2.10.

Um, J., Lightsey, E.G. (2001). Attitude Determination for SOAR Experiment, Navigation - Journal of The Institute of Navigation, 48(3): 181-194.

Sutton, E.(1997). Optimal Search Space Identification for Instantaneous Integer Cycle Ambiguity Resolution, Proceedings of ION GPS-97 (pp. 313-322), Alexandria, VA: Institute of Navigation. 
Ward, L.M., Axelrad, P. (1996). A Combination Filter for GPS-Based Attitude and Baseline Estimation, Proceedings of ION GPS-96 (pp.1047-1061), Alexandria, VA: Institute of Navigation.

Wahba, G. (1965). A Least Squares Estimate of Satellite Attitude, SIAM Review, 7(3), 409.

Wertz, J.R. (1984). Spacecraft Attitude Determination and Control D. Reidel, Dordrecht, The Netherlands, 764.

Ziebart, M., Cross, P. (2003). LEO GPS Attitude Determination Algorithm for a Micro-satellite Using Boomarm deployed Antennas, GPS Solutions, 6(4), 242-256. 
Figure 1. Flowchart of the proposed GPS attitude determination algorithm

Figure 2. The 2D and 3D plots of the pitch-yaw ambiguity resolution function

Figure 3. Trimble TANS Vector GPS receiver with four antennas

Figure 4. The line bias solution and the error of the attitude solution in the field test

Figure 5. GPS antenna mounting and baseline configuration in actual flight experiments

Figure 6. The 3D trajectory of the flight experiment and baseline bias solution

Figure 7. GPS attitude solution (roll, pitch and yaw) vs. GPS time

Figure 8. Difference between GPS attitude solution and output of IMU 


\begin{tabular}{llll}
\hline \hline Line Biases & $\beta_{1}$ & $\beta_{2}$ & $\beta_{3}$ \\
\hline Winter (23/12/1998) & -0.200 & -0.083 & 0.064 \\
Summer (23/07/1999) & -0.149 & -0.071 & 0.074 \\
\hline \hline
\end{tabular}

Table 1. Comparison of the line bias solution in different seasons (in cycles)

\begin{tabular}{llll}
\hline \hline Error of attitude solution & $\Delta$ Roll & $\Delta$ Pitch & $\Delta$ Yaw \\
\hline Standard deviation & 0.18 & 0.30 & 0.41 \\
Minimum & -0.56 & -0.76 & -2.17 \\
Maximum & 0.88 & 0.77 & 1.45 \\
\hline \hline
\end{tabular}

Table 2. Evaluation of attitude solution error in the field test (in degrees)

\begin{tabular}{ccc}
\hline \hline Error of angles & Average & Standard deviation \\
\hline$\Delta$ Roll & 0.012 & 0.16 \\
$\Delta$ Pitch & -0.056 & 0.13 \\
$\Delta$ Yaw & -0.054 & 0.23 \\
\hline \hline
\end{tabular}

Table 3. The average and standard deviation of the discrepancies between GPS and IMU solutions (unit: degrees) 